\title{
Nonlinear tsunami generation mechanism
}

\author{
M. A. Nosov and S. N. Skachko \\ Physics of Sea and Inland Waters Chair, Faculty of Physics, M. V. Lomonosov Moscow State University, Vorobjevy Gory, \\ 119899 Moscow, Russia
}

Received: 11 July 2001 - Accepted: 23 August 2001

\begin{abstract}
The nonlinear mechanism of long gravitational surface water wave generation by high-frequency bottom oscillations in a water layer of constant depth is investigated analytically. The connection between the surface wave amplitude and the parameters of bottom oscillations and source length is investigated.
\end{abstract}

\section{Introduction}

If during an underwater earthquake the frequency of vertical bottom oscillations is greater than $\sqrt{g / H}$ and the oscillations are not accompanied by residual displacement, then an effective excitement of surface gravity waves (tsunami) is impossible (Nosov, 1999). This effect is a result of linear theory. However, strong underwater earthquakes are certainly accompanied by bottom oscillations of significant velocity magnitude; thus linear theory becomes inapplicable and nonlinear phenomena cannot be neglected. Here, we consider long gravitational wave generation as a result of nonlinear water layer fast oscillations "rectification". Our goal is to study the relationship between the long gravitational wave amplitude and bottom oscillations parameters.

\section{Basic mathematical model}

Let us consider an ideal incompressible homogeneous fluid layer of constant depth $H$ in the gravity field. The Cartesian coordinate system $O X Z$ origin finds itself at the unperturbed free surface and $O Z$-axis is oriented vertically upward. It is assumed that fluid velocity consists of oscillating (fast) and time averaged (slow) terms:

$u^{\sum}(x, z, t)=u(x, z) \cos (\omega t)+U(x, z, t)$,

$w^{\sum}(x, z, t)=w(x, z) \cos (\omega t)+W(x, z, t)$,

where $u$ and $w$ are horizontal and vertical fluid velocity components.

Correspondence to: M. A. Nosov (psiwc47@phys.msu.su)
Substituting expressions (1) and (2) into the left part of the Euler equations and averaging these equations in time we obtained:

$$
\begin{aligned}
\overline{\frac{\partial u^{\Sigma}}{\partial t}}+u^{\Sigma} \frac{\partial u^{\Sigma}}{\partial x}+w^{\Sigma} \frac{\partial u^{\Sigma}}{\partial z} \\
=\frac{\partial U}{\partial t}+U \frac{\partial U}{\partial x}+W \frac{\partial U}{\partial z}-f_{x},
\end{aligned}
$$

$$
\begin{aligned}
& \overline{\frac{\partial w^{\Sigma}}{\partial t}+u^{\Sigma} \frac{\partial w^{\Sigma}}{\partial x}+w^{\Sigma} \frac{\partial w^{\Sigma}}{\partial z}} \\
& \quad=\frac{\partial W}{\partial t}+U \frac{\partial W}{\partial x}+W \frac{\partial W}{\partial z}-f_{z},
\end{aligned}
$$

where

$$
\begin{aligned}
& f_{x}(x, z)=-\frac{1}{2}\left[u(x, z) \frac{\partial u(x, z)}{\partial x}+w(x, z) \frac{\partial u(x, z)}{\partial z}\right], \\
& f_{z}(x, z)=-\frac{1}{2}\left[u(x, z) \frac{\partial w(x, z)}{\partial x}+w(x, z) \frac{\partial w(x, z)}{\partial z}\right] .
\end{aligned}
$$

Euler equations' non-linearity produced additional terms in the governing equations for the time averaged flow. These terms $(3,4)$ can be considered as $x$ and $z$ external mass force components.

\section{Auxiliary linear problem}

In order to calculate the external mass force it is necessary to define the velocity field in the fluid layer. Let us define this field as a linear response of an ideal fluid to bottom oscillations $\eta(x, t)$. We can consider an auxiliary linear problem in terms of the fluid velocity potential $F(x, z, t)$ :

$F_{x x}+F_{z z}=0$

$F_{t t}=-g F z, \quad z=0$,

$F_{z}=\eta_{t}, \quad z=-H$ 

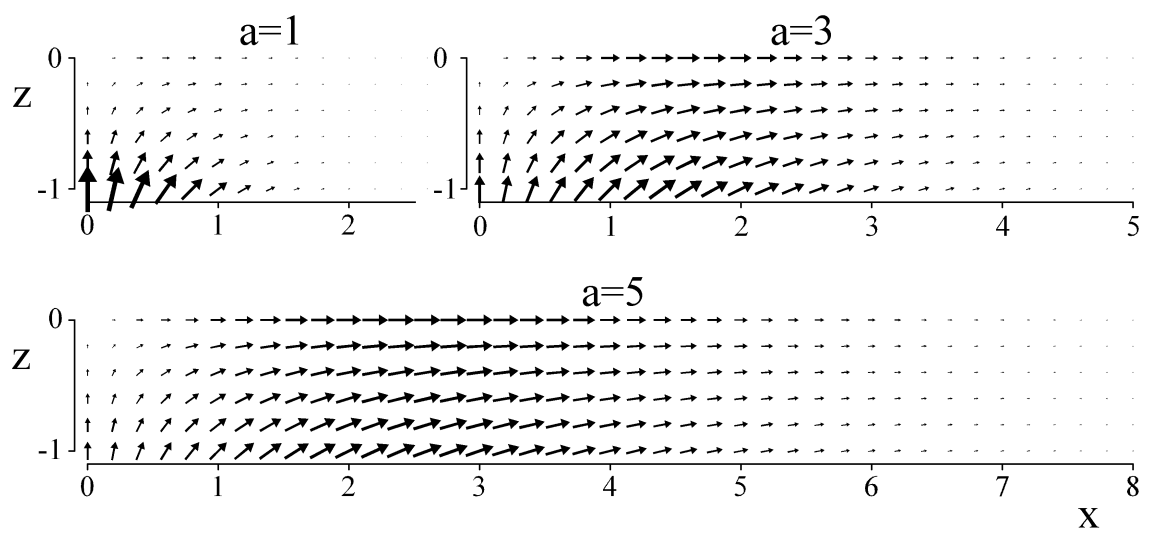

Fig. 1. Examples of spatial distribution of the mass force $\eta_{1}(x)$ (from formula (9), $a=1,3$ and 5).

The potential Eq. (5) with boundary conditions on surface (6) and bottom (7) was solved using the standard method of separation of variables. A general solution of this problem in terms of Laplas and Fourier expansions is:

$$
\begin{gathered}
F(x, z, t)=-\frac{1}{4 \pi^{2} i} \int_{s-i \infty}^{s+i \infty} d p \int_{-\infty}^{\infty} d k \\
\frac{p \exp (p t-i k x) \operatorname{ch}(k z)\left[g k-p^{2} t h(k z)\right]}{k \operatorname{ch}(k H)\left[g k \operatorname{th}(k H)+p^{2}\right]} G(p, k),
\end{gathered}
$$

where

$G(p, k)=\int_{0}^{\infty} d t \int_{-\infty}^{\infty} d x \exp (-p t+i k x) \eta(x, t)$

The velocity components can be expressed in terms of potential:

$u(x, z, t)=\frac{\partial F}{\partial x}, w(x, z, t)=\frac{\partial F}{\partial z}$.

We assume the following function for bottom oscillations:

$\eta(x, t)=\eta_{i}(x) \theta(t) \sin (w t), \quad i=1,2$

$\eta_{1}(x)=\eta_{0} \exp \left(-x^{2} a^{-2}\right)$

$\eta_{2}(x)=\left\{\begin{array}{cc}\eta_{0}, & |x| \leq b, \\ \eta_{0} c^{-1}(b-|x|)+1, & b<|x| \leq b+c, \\ 0, & |x|>b+c,\end{array}\right.$

where $\eta_{0}$ and $\omega$ are the amplitude and the frequency of bottom oscillations, respectively, $\theta$ is the Heaviside step function.

We introduce nondimension variables (the superscript “*” will be omitted hereafter)

$k^{*}=H k, t^{*}=t(g / H)^{1 / 2}, \omega^{*}=\omega(H / g)^{1 / 2}$,

$\left\{x^{*}, z^{*}, a^{*}, b^{*}, c^{*}\right\}=H^{-1}\{x, z, a, b, c\}$,

$\left\{u^{*}, w^{*}\right\}=\{u, w\} /\left(\eta_{0} \omega\right)$.
The final formulae for the fluid velocity components are as follows:

$$
\begin{aligned}
& u(x, z, t)=\frac{1}{\pi} \int_{0}^{\infty} d k \frac{\sin (k x) \operatorname{ch}(k z) X_{i}(k)}{\operatorname{ch}(k)\left(p_{0}^{2}-\omega^{2}\right)} \\
& \times\left\{\cos (\omega t)\left[k+\omega^{2} t h(k z)\right]-\cos \left(p_{0} t\right)\left[k+p_{0}^{2} t h(k z)\right]\right\} \\
& w(x, z, t)=-\frac{1}{\pi} \int_{0}^{\infty} d k \frac{\cos (k x) \operatorname{ch}(k z) X_{i}(k)}{\operatorname{ch}(k)\left(p_{0}^{2}-\omega^{2}\right)} \\
& \times\left\{\cos (\omega t)\left[k t h(k z)+\omega^{2}\right]-\cos \left(p_{0} t\right)\left[k t h(k z)+p_{0}^{2}\right]\right\}
\end{aligned}
$$

where

$p_{0}^{2}=k \operatorname{th}(k)$,

$X_{i}(k)=\int_{-\infty}^{+\infty} d x \exp (i k x) \eta_{i}(x)$.

Numerical analysis of expressions (11), (12) gives us the basis to state that in the frequency range $\omega>2 \pi \sqrt{g / H}$ each point of fluid oscillates harmonically in accordance with expressions (1), (2). Now, since we have explicit expressions for the functions $u(x, z)$ and $w(x, z)$, it is easy to calculate the force components $f_{x}$ and $f_{z}$ using formulae (3) and (4).

The force spatial distribution examples are shown in Fig. 1. They were calculated for the bottom oscillation amplitude distribution $\eta_{1}(x)$ for different source lengths. It is evident from the figure that such force distribution can lead to the generation of long gravitational wave (tsunami). As the source length increases the vertical component of the force becomes negligible in comparison with the horizontal one. The horizontal size of the tsunami source usually exceeds the ocean depth significantly; so the linear shallow water theory can be applied in order to estimate gravitational waves excited by the mass force.

\section{Long wave generation}

The linear shallow water theory equations, taking into account effect of the horizontal force $f(x, t)$

$\frac{\partial U}{\partial t}=-g \frac{\partial \xi}{\partial x}+f(x, t)$, 


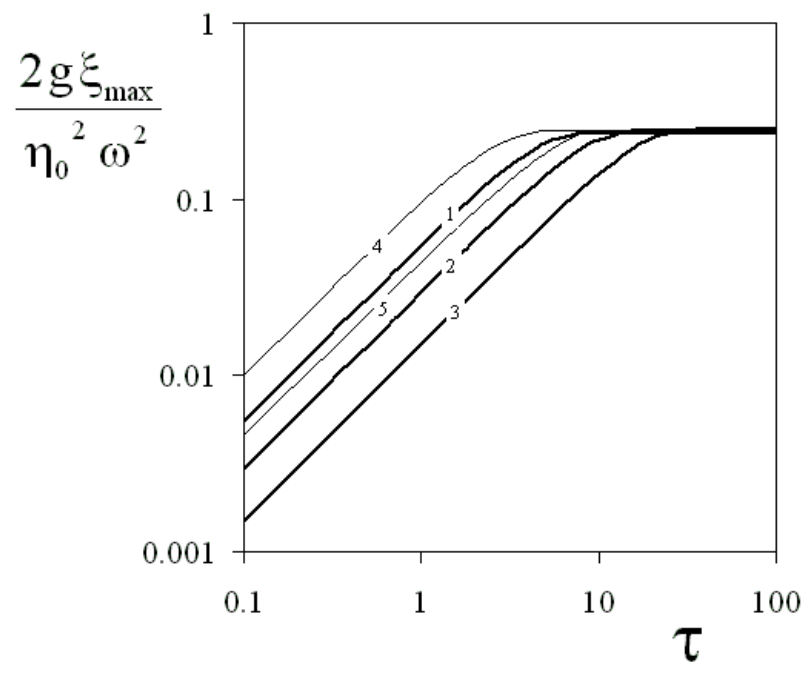

Fig. 2. The maximum amplitude of the long wave as a function of bottom oscillation duration. Curves 1,2, 3 correspond to $\eta_{1}(x)$ from formula (9), $a=5,10$ and 20; curves 4 and 5 correspond to $\eta_{2}(x): b=2, c=3$ (4), and $b=1, c=9$ (5).

$\frac{\partial \xi}{\partial t}+H \frac{\partial U}{\partial x}=0$

are reduced by a traditional approach to a nonhomogeneous wave equation; the equation is written in dimensionless form:

$\frac{\partial^{2} \xi}{\partial x^{2}}-\frac{\partial^{2} \xi}{\partial t^{2}}=\frac{H}{g} \frac{\partial f}{\partial x}$,

where $\xi$ is a free surface displacement from the equilibrium and $\xi$ and $f$ in Eq. (13) are dimensional quantities. The analytical solution of this equation is well-known:

$\xi(x, t)=\frac{H}{2 g} \int_{0}^{t} d \sigma \int_{x-(t-\sigma)}^{x+(t-\sigma)} \frac{\partial f}{\partial \varsigma} d \varsigma$.

Let us consider bottom oscillations of constant amplitude and frequency. Duration of the oscillations is $\tau$. The function $f(x, t)$ can be written in the following form:

$f(x, t)=f(x)[\theta(t)-\theta(t-\tau)]$.

Substituting (15) in (14) and integrating over $d \xi$ we obtained

$$
\begin{gathered}
\xi(x, t)=-\frac{H}{2 g} \int_{0}^{t}[\theta(\sigma)-\theta(\sigma-\tau)] \\
{[f(x+(t-\sigma))-f(x-(t-\sigma))] d \sigma .}
\end{gathered}
$$

We can define function $f(x)$ using formula (3) as follows $f(x)=f_{x}(x,-0.5)$.

\section{Discussion of results}

Using Eq. (16) we obtained free surface displacements generated by a nonlinear mechanism. Figure 2 shows the max- imum displacement amplitude $\xi_{\max }$ plotted as a function of the oscillations duration $\tau$. The maximum amplitude rises monotonically until value 0.25 as the duration goes up. Neither source horizontal size nor the spatial distribution $\eta_{i}(x)$ alter this dependence by much. In all cases the maximum amplitude reaches the same value 0.25 at large $\tau$.

This nonlinear effect can be interpreted in the following way. The amplitude of fluid particle oscillations at a given point decreases as the point moves away from the source. This is why fluid particles after the oscillation period did not return exactly to the previous position. Thus fluid is "extruded" from the more intensive oscillations areas. This is a cause of the long gravity wave formation. The amplitude of such wave depends on the spatial distribution of bottom oscillations, on the oscillating velocity amplitude $\eta_{0} \omega$, and on the duration $\tau$.

The data shown in Fig. 2 allows one to estimate a contribution of the nonlinear mechanism in the tsunami amplitude. For the source parameters $H=1 \mathrm{~km}, a=10 \mathrm{~km}$ (spatial distribution $\eta_{1}$ ) bottom oscillations with velocity amplitude of $10 \mathrm{~m} / \mathrm{s}$ generate a wave of $0.8 \mathrm{~m}$ height during $60 \mathrm{~s}$.

It should be noted that the compressible fluid theory has to be employed in case of high frequency bottom oscillations. It imposes certain restrictions on the application of the results received above. Novikova and Ostrovsky (1982) also studied the nonlinear tsunami generation mechanism; they took into account water compressibility. However, in order to define tsunami parameters, a hypothetical acoustic field was used; thus a connection between bottom motions and the gravitational long wave was not established. In contrast to the paper by Novikova and Ostrovsky, (1982), in our study the maximum tsunami amplitude was calculated as a bottom oscillation parameters function.

In the future, we certainly plan to take into account water compressibility. The necessary linear models have been developed by us earlier (Nosov and Sammer, 1998; Nosov, 1999, 2000).

Acknowledgement. This work was supported by the Russian Foundation for Basic Research, project 01-05-64547.

\section{References}

Nosov, M. A.: Generation of tsunami by oscillations of a sea floor section, Moscow University Physics Bulletin, 47, 1, 110-112, 1992.

Nosov, M. A.: Tsunami generation in a compressible ocean by vertical bottom motions, Izvestiya, Atmospheric and Oceanic Physics, 36, 5, 718-726, 2000.

Nosov, M. A.: Tsunami generation in compressible ocean, Phys. Chem. Earth (B), 24, 5, 437-441, 1999.

Nosov, M. A. and Sammer, K.: Tsunami excitation by a moving bottom displacement in compressible water, Moscow University Physics Bulletin, 53, 6, 67-70, 1998.

Novikova, L. E. and Ostrovsky, L. A.: On an acoustic mechanism of tsunami wave generation, Oceanology, 22, 5, 693-697, 1982. 